\title{
System Size Effects on Gyrokinetic Turbulence
}

\author{
B. F. McMillan, X. Lapillonne, S. Brunner, and L. Villard \\ Centre de Recherches en Physique des Plasmas, Association Euratom-Confédération Suisse, \\ Ecole Polytechnique Fédérale de Lausanne, PPB, 1015 Lausanne, Switzerland \\ S. Jolliet \\ Japan Atomic Energy Agency, Higashi-Ueno-6-9-3, Taitou, Tokyo 110-0015, Japan \\ A. Bottino, T. Görler, and F. Jenko \\ Max Planck Institut für Plasmaphysik, Boltzmannstrasse 2, D-85748 Garching, Germany
}

(Received 30 June 2010; published 4 October 2010)

\begin{abstract}
The scaling of turbulence-driven heat transport with system size in magnetically confined plasmas is reexamined using first-principles based numerical simulations. Two very different numerical methods are applied to this problem, in order to resolve a long-standing quantitative disagreement, which may have arisen due to inconsistencies in the geometrical approximation. System size effects are further explored by modifying the width of the strong gradient region at fixed system size. The finite width of the strong gradient region in gyroradius units, rather than the finite overall system size, is found to induce the diffusivity reduction seen in global gyrokinetic simulations.
\end{abstract}

The ultimate purpose of gyrokinetic simulations of tokamaks is to predict the transport properties of fusion reactors. This is often restricted to the less ambitious goal of predicting how the transport properties scale with system size, so that the properties of a reactor can be estimated using measurements of transport in smaller tokamaks.

Most of the scaling studies to date [1,2] have used the Cyclone model equilibrium [3], based loosely on an experiment on the DIII-D tokamak [4]. The Cyclone parameters have been used as a standard case for benchmarking gyrokinetic codes, and as a baseline case for parameter scans with both global and flux-tube codes, with a variety of approximations taken for the background geometry and magnetic field. The heat flux is quite sensitive to minor model changes because the baseline parameters are relatively close to marginal nonlinear stability. As a result, both finite system size effects and geometrical assumptions can make a relatively large difference to the predicted heat diffusivity (up to a factor of 2), so that direct comparisons between code results are exacting.

In this Letter we reexamine the effects of global system size and temperature gradient profiles on the heat diffusivity. To improve the confidence in the final results we perform simulations with two gyrokinetic codes with very different numerical methods, which have been carefully benchmarked, both independently [5-7] and against each other [8]. The global-GENE code [6,7] is a generalization of the flux-tube Eulerian GENE [9] code to global geometry. ORB5 [5] is a global particle-in-cell code.

For the equilibrium description, we choose the circular concentric model of Refs. [5,10], which is convenient for numerical studies as it provides simple expressions for geometrical and magnetic field quantities, although it is not a true MHD equilibrium. A flux-tube gyrokinetic analysis finds linear growth rates and a heat diffusivity for this model which are quite close to those obtained using an appropriate MHD equilibrium. This is not the case for typical implementations of the $s$ - $\alpha$ model, where the assumption that the geometrical poloidal angle is a straight field line coordinate leads to inconsistencies between geometric quantities and the field strength which are first order in the inverse aspect ratio [10].

Ion temperature gradient (ITG) turbulence is driven on the scale of the ion gyroradius, $\rho_{i}$, but a related quantity, the ion sound radius $\rho_{s}$, is typically used to parameterize the turbulent length scale. The value of the parameter $1 / \rho^{*}=a / \rho_{s}$, where $a$ is the tokamak minor radius, is thus a useful dimensionless measure of system size. In order to evaluate how global effects influence turbulence levels, a $\rho^{*}$ scan is carried out using the global-GENE and ORB5 codes, for $1 / \rho^{*} \in[70,140,180,280,560]$, and the results are compared to a GENE flux-tube simulation. The Cyclone parameters are $T=Z T_{e}, a / R=0.36, \kappa_{n}=2.23$, $\kappa_{T}=6.96$, with $T$ and $T_{e}$ the ion and electron temperatures, respectively, $R$ the major radius, and $\kappa_{n}$ and $\kappa_{T}$ the logarithmic density and temperature gradient at midradius, respectively. Electrons are modeled as an adiabatic species, and the simulations are performed in the electrostatic limit. We choose a safety factor profile $q(r)=(0.854+$ $\left.2.184 r^{2}\right) /\left[1-(r a / R)^{2}\right]^{1 / 2}$, where $r$ is the distance from the magnetic axis, normalized to the minor radius. For this profile, $q(0.5)=1.4$ and $s(0.5)=(r / q) d q / d r=0.8$ to match the Cyclone parameters at midradius. Two very 
similar functional forms of the initial temperature gradient profile are used. For the first,

$$
\frac{R}{a T} \frac{d T}{d r}=-\kappa_{T}\left[1-\cosh ^{-2}\left(\frac{r_{-}}{\Delta_{T}}\right)-\cosh ^{-2}\left(\frac{r_{+}}{\Delta_{T}}\right)\right]
$$

for $r \in\left[r_{0}-\Delta_{r} / 2, r_{0}+\Delta_{r} / 2\right]$, and zero elsewhere, with $r_{ \pm}=r-r_{0} \pm \Delta_{r} / 2$. The other functional form is

$$
\frac{R}{a T} \frac{d T}{d r}=-\frac{\kappa_{T}}{2}\left[\tanh \left(\frac{r_{-}}{\Delta_{T}}\right)-\tanh \left(\frac{r_{+}}{\Delta_{T}}\right)\right] .
$$

The ion density profiles are defined using the same functional form, with the scale factor $\kappa_{n}$ replacing $\kappa_{T}$. Both ORB5 and GENE were run with $r_{0}=0.5$, with $\Delta_{r}=0.8$ and 0.7 for ORB5 and GENE, respectively, so that $R / L_{T}=$ $-R d \ln (T) / d r$ is constant over most of the minor radius and equal to $\kappa_{T}$. In this section, GENE uses the first functional form, and ORB5 the second. ORB5 uses a canonical Maxwellian to define the background distribution, rather than a local Maxwellian, so the reconstructed temperature profiles differ slightly from nominal values (the bracketing described below corrects for this effect).

For the GENE code, 33 toroidal modes were examined, with $k_{y} \in[0.048,1.6]$ for $\rho^{*}=1 / 180$, and similar $k_{y}$ range for other $\rho^{*}$. For the baseline case at $\rho^{*}=1 / 180$, the number of grid points is 150,64 , and 16 in the radial, binormal, and field line direction, respectively. For different $\rho^{*}$, the number of radial grid points is scaled to keep the resolution constant in units of ion gyroradius. 64 grid points are used in $v_{\|}$, and 16 in $\mu$. The simulation domain is restricted to a radial domain $0.1<r<0.9$, and, for $\rho^{*}=$ $1 / 180$, a $1 / 3$ wedge of the full torus. The values of the hyperdiffusion terms, added for stability reasons, can be found in Ref. [6].

For ORB5, the $\rho^{*}=1 / 180$ simulations were performed with a field grid of $128 \times 512 \times 256$ points in the radial, poloidal, and toroidal directions, respectively. 45 toroidal modes were kept, and the simulation domain is a $1 / 2$ wedge of the full torus from $0<r<1.320 \times 10^{6}$ markers were used, and a Krook operator with a coefficient $0.0035 c_{s} / a, \leqslant 2.5 \%$ of the peak linear growth rate, for the range of $\rho^{*}$ considered (this is at least a factor of 4 smaller than suggested by convergence requirements [11]). For different $\rho^{*}$, the number of field grid points in each direction is scaled proportional to $1 / \rho^{*}$, the fractional size of the wedge is scaled like $\rho^{*}$, and the number of markers is scaled according to $1 / \rho^{*}$, to keep the number of markers per mode constant.

Dirichlet boundary conditions are used in GENE for the electrostatic potential at the inner and outer radial points, whereas ORB5 imposes Dirichlet conditions only at the outer boundary. The boundary conditions on the distribution function are also slightly different, and are described in detail in Refs. [5,6]. The restriction to a wedge of the torus of roughly constant size in gyroradius units is motivated by earlier convergence studies.
Both codes use a heat source proportional to the temperature deviation $\delta T=T(r, t)-T(r, 0)$; as a consequence, the steady state temperature gradient differs somewhat from the initial temperature gradient, in a way which cannot accurately be estimated prior to a simulation run. In order to compute the heat diffusivity over this $\rho^{*}$ scan at a desired temperature gradient, two simulations were carried out for each $\rho^{*}$ value, with $\kappa_{T}=7.1$ and $\kappa_{T}=7.5$. This gives us a pair of values for the final temperature gradients which closely bracket the target temperature gradient $R / L_{T}=7.0$. The diffusivity $\chi_{i}$ corresponding to the target gradient is then computed using linear interpolation. In Fig. 1, the resulting heat diffusivities $\chi_{i}\left(R / L_{T i}=7\right)$ obtained with both global-GENE and ORB5 are shown in gyro-Bohm units $\left(\chi_{\mathrm{GB}}=\rho_{s}^{2} c_{s} / a\right)$ as a function of $\rho^{*}$, and the results are compared to a GENE fluxtube simulation.

The relative difference between the flux-tube and global heat diffusivity is below $10 \%$ for $1 / \rho^{*} \gtrsim 280$, confirming the qualitative results of Refs. $[2,12]$ that the global gyrokinetic codes reproduce the flux-tube diffusivities in the limit $\rho^{*} \rightarrow 0$, where the gyro-Bohm scaling is prescribed. Gyro-Bohm diffusivity scaling is expected in this limit based on a mixing length argument, in which eddies on turbulence scale, $\sim \rho_{s}$, are able to transport energy across an eddy width on the time scale of the driving instability, $\sim a / c_{s}$. The minor radius enters the time scale because, for fixed geometry, the growth rate is dependent on the field line curvature and inverse gradient lengths, which scale with $1 / a$.

The ORB5 and global-GENE results show a similar $\rho^{*}$ dependence, as well as an excellent overall agreement, within $10 \%$, between the two codes. An estimate of the

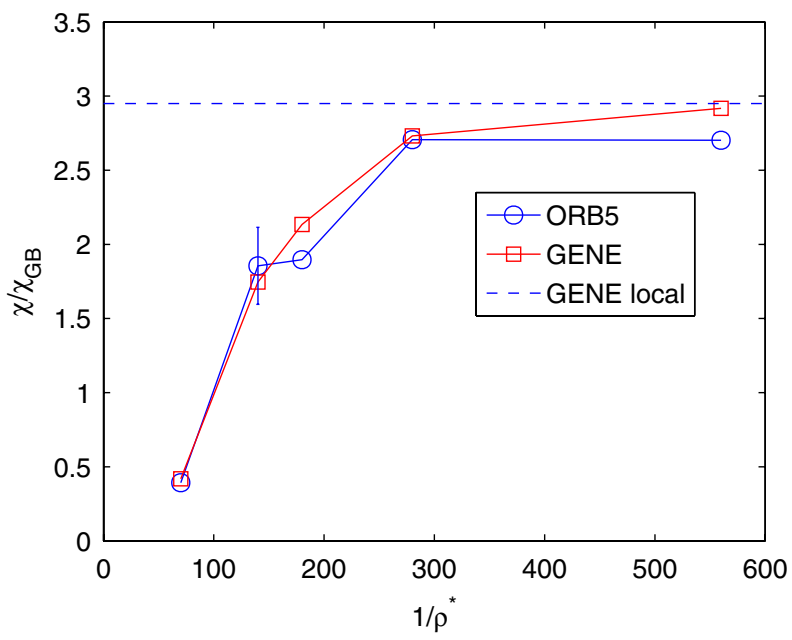

FIG. 1 (color online). Ion heat diffusivity $\chi_{i} / \chi_{\mathrm{GB}}\left(\chi_{\mathrm{GB}}=\right.$ $\left.\rho_{s}^{2} c_{s} / a\right)$ obtained with global-GENE and ORB5 as function of $1 / \rho^{*}$. A radial window, $r \in[0.4,0.6]$ is used, and a time average over $t c_{s} / R \in[210,450]$. The results are compared with the corresponding GENE flux-tube simulation. The error bar represents simulation-to-simulation variability. 
simulation-to-simulation variability of the measured $\chi_{i}$ due to turbulent variation has been obtained for the $1 / \rho^{*}=$ 180 case by carrying out three independent ORB5 simulations with different initial conditions [11]. The diffusivities predicted by the two codes are essentially within the error bars, despite the very different numerical methods and a somewhat different set of approximations. In the $\rho^{*} \rightarrow 0$ limit, both diffusivity curves asymptote to $\chi_{i} / \chi_{\mathrm{GB}} \simeq$ 2.8-2.9, similar to the value found in flux-tube simulations using the circular model equilibrium $\left(\chi_{i} / \chi_{\mathrm{GB}}=3.0\right)$ or a MHD equilibrium $\left(\chi_{i} / \chi_{\mathrm{GB}}=3.3\right)$ [10].

Results of the GYRO code [2] using an $s-\alpha$ approximation, can be compared against the set of results of the GTC code [13] with finite aspect ratio effects "removed" (although presumably some finite aspect ratio effects, like magnetic trapping, were kept) to try to reproduce the $s-\alpha$ results. These GTC results appear to asymptote to at least $\chi_{i} / \chi_{\mathrm{GB}} \sim 2.5$ in the flux-tube limit. On the other hand, the global GYRO results in Ref. [2] converge towards a value of $\chi_{i} / \chi_{\mathrm{GB}} \simeq 1.9$ in the limit $\rho^{*} \rightarrow 0$.

The circular analytical equilibrium used for the GTC $\rho^{*}$ scaling runs of Ref. [1], yields linear growth rates close to those using the numerical MHD equilibria [12], and appears to be similar to the equilibrium used by GENE and ORB5. Diffusivities from the GTC $\rho^{*}$ scans of Ref. [1] converge towards $\chi_{i} / \chi_{\mathrm{GB}} \simeq 3.4$ in the limit $\rho^{*} \rightarrow 0$, which is in relatively good agreement with the present results $\chi_{i} / \chi_{\mathrm{GB}} \simeq 2.8-2.9$. This agreement occurs despite notable differences between these two studies, including the use of shorter simulations in Ref. [1].

In the $s-\alpha$ approximation the geometry specification is inconsistent with the magnetic field specification. However, in their manipulations of the gyrokinetic equations, code authors often assume that the magnetic field and geometry are consistent. The final equations used might, for example, include terms like the divergence of the magnetic field, which is nonzero in the $s$ - $\alpha$ model, or these might have been explicitly set to zero by code authors, given that physical fields are divergence free. The results of using the $s-\alpha$ approximation will thus not only be incorrect, but incorrect in a way which may vary from code to code, so that results are not easily reproducible. Many flux-tube codes using the $s$ - $\alpha$ model find a diffusivity of $\chi_{i} / \chi_{\mathrm{GB}} \sim 1.7$ [3] for the Cyclone test case, substantially different from the value $\chi_{i} / \chi_{\mathrm{GB}} \sim 3.3$ found using an MHD equilibrium, or 3.0 found using the circular concentric model [10]. The use of the $s$ - $\alpha$ model may also explain why the results of Ref. [2] differ from ours. We suggest that future benchmarks be run using consistent equilibria.

For a fixed local temperature gradient, the shape of the temperature profile is known to modify turbulence intensity [2]. To quantify this effect, two extra pairs of simulations were run at $\rho^{*}=1 / 280$, with the normalized width $\Delta_{r}$ of the strong temperature region set to $\sim 0.4$ and $\sim 0.2$,

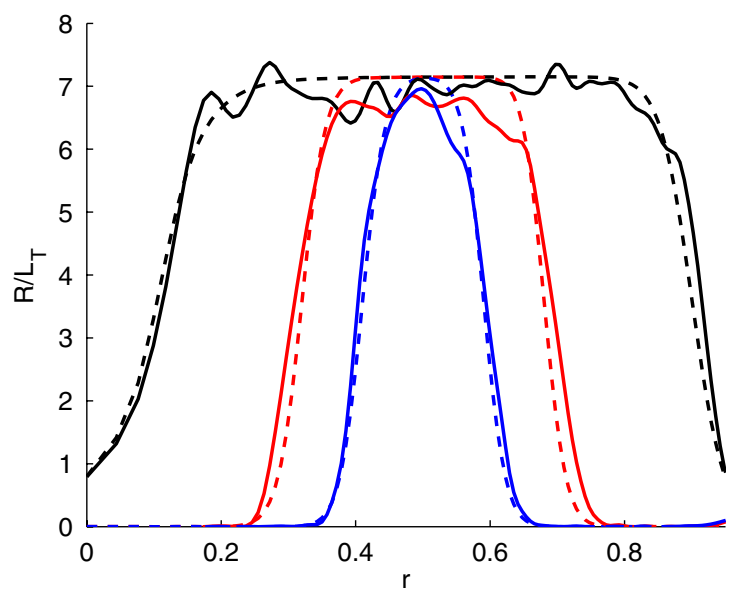

FIG. 2 (color online). Logarithmic temperature gradients plotted against radius for simulations with $\rho^{*}=1 / 280$, and $\Delta_{r}=$ $0.2,0.4,0.8$, in order of increasing width of the plotted peak. The dashed lines show initial gradients, and the solid lines show the late time average over the last half of the simulation time.

approximately one-half and one-quarter of the values in the previous section (see Fig. 2). We again use pairs of simulations with slightly different nominal temperature gradients, to bracket the desired final temperature gradient. Here, both codes use Eq. (2) for temperature and density gradient profiles, but parameters are otherwise identical to the simulations described above. The mean flux levels of these simulations, together with those of the previous section, are plotted in Fig. 3, against the measured width of the strong gradient region $\rho_{\text {eff }}^{*}=\rho^{*} / \Delta_{r}$, in units of gyroradius. The width $\Delta_{r}$ is the full width at half maximum of the initial reconstructed temperature gradient profile; because the heating operator is quite effective at maintaining these profiles, using late time values makes very little

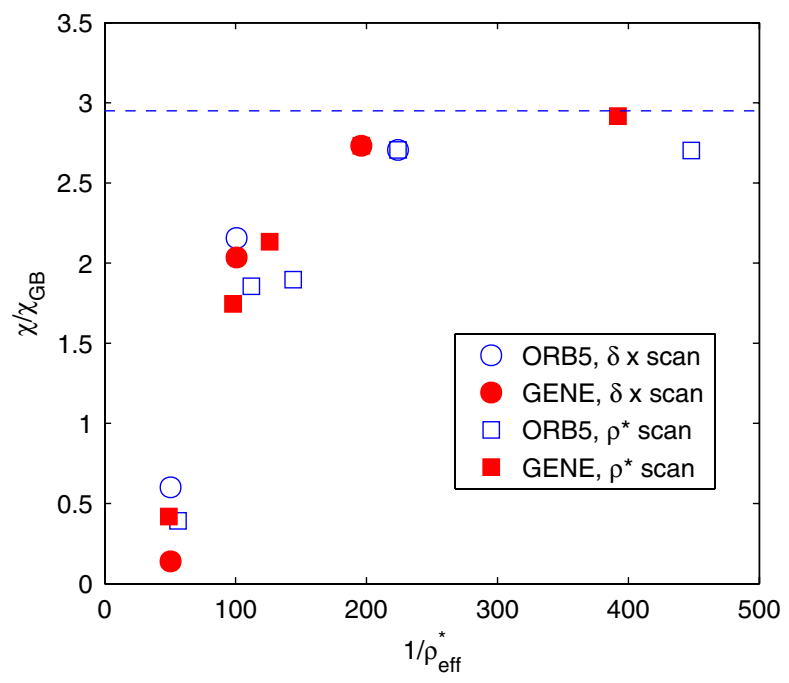

FIG. 3 (color online). Average flux levels $r \in[0.4,0.6]$ for $t \in$ $[150,410] R / c_{s}$ versus the width of the strong gradient region, $1 / \rho_{\mathrm{eff}}^{*}$, in gyroradius units. 
difference. The good agreement between the data points in Fig. 3 suggests that the variation in diffusivity levels in the $\rho^{*}$ scan is a result of the changing width of the strong gradient region (in units of gyroradius) rather than the system size $\rho^{*}$ itself. Note that several of the data points in Fig. 3 have very similar $\rho_{\text {eff }}^{*}$, and levels of diffusivity, but very different $\rho^{*}$. Any influence due to the overall system size $\rho^{*}$ is seen to be small compared to that of $\rho_{\text {eff }}^{*}$. The diffusivity reduction at large $\rho_{\text {eff }}^{*}$ would thus be expected to be important not only in small tokamaks, but also in transport barriers, where strong gradients appear over a small proportion of the minor radius.

The radial window used for flux averaging becomes comparable to the width of the strong gradient region for the extreme case $\Delta_{r}=0.2$. However, the measured diffusivity is not very sensitive to the choice of the averaging window: if the width of the flux averaging window is reduced proportionately to $\Delta_{r}$, the results are essentially unchanged.

Because changing $\rho^{*}$ does not appear to substantially modify the diffusivities, for fixed $\rho_{\text {eff }}^{*}$, the mechanisms responsible for the flux reduction should exist in the fluxtube-like limit where $\rho^{*} \rightarrow 0$, but $\rho_{\text {eff }}^{*}$ is held constant. This limit is different from the usual flux-tube limit only through the choice of a modified initial temperature profile, and the use of a heat source term to maintain this temperature gradient profile; the temperature, shear, and density would be constant across the radial domain, as in a standard flux-tube simulation.

Profile shearing is a form of phase mixing which occurs because the local mode frequency $\omega(r)$ (calculated for a homogeneous plasma) has a nonzero radial derivative, so that radial phase mixing occurs, and modes tend to secularly become tilted with time. This flux reduction mechanism is analogous to that due to sheared zonal flows. At fixed $\rho_{\mathrm{eff}}^{*}$, the variation of $\omega(r)$ over the strong gradient region, where the logarithmic temperature gradient is nearly constant, depends on $\rho^{*}$, but the results are clearly unaffected by this variation: profile shearing cannot be causing the diffusivity reduction at large $\rho_{\text {eff }}^{*}$.

Some nonlocality is expected because turbulent eddies have radial extent of $\sim 20$ gyroradii, so sense an interval of the minor radius, but this is not enough to explain the significant flux reduction seen at $\rho_{\text {eff }}^{*}=1 / 120$, where the edge of the strongly driven region is 60 gyroradii from the center of the averaging window. Information appears to propagate over several eddy lengths (or turbulence decorrelation lengths). The diffusion of turbulent intensity, or "turbulence spreading" [14], is one possible way to explain strong nonlocal effects. The presence of larger scale structures, like zonal flows, or avalanches [15] is also expected to lead to substantial nonlocality.
To summarize, for the Cyclone test parameters, we confirm that the diffusivities of finite plasmas approach the flux-tube limit from below in the large system size limit, in agreement with Ref. [2]. Quantitatively, we find an asymptotic diffusivity of $\chi_{i} / \chi_{\mathrm{GB}} \simeq 2.8-2.9$. This substantially differs from the value found in Ref. [2], which used an inconsistent $s$ - $\alpha$ equilibrium model, but in rough agreement with the results of Ref. [1], which used a more realistic equilibrium. Moreover, we find that the reduction of flux at finite system size is largely the result of the finite width of the unstable region in gyroradius units, rather than the overall tokamak size.

This work was supported in part by the Swiss National Science Foundation, a grant from the Swiss National Supercomputing Centre-CSCS, and by the EPFL BlueGene facility.

[1] Z. Lin, S. Ethier, T. S. Hahm, and W. M. Tang, Phys. Rev. Lett. 88, 195004 (2002).

[2] J. Candy, R. E. Waltz, and W. Dorland, Phys. Plasmas 11, L25 (2004).

[3] A. M. Dimits, G. Bateman, M. A. Beer, B. I. Cohen, W. Dorland, G. W. Hammett, C. Kim, J.E. Kinsey, M. Kotschenreuther, and A. H. Kritz et al., Phys. Plasmas 7, 969 (2000).

[4] C. M. Greenfield, J.C. DeBoo, T.H. Osborne, F. W. Perkins, M.N. Rosenbluth, and D. Boucher, Nucl. Fusion 37, 1215 (1997).

[5] S. Jolliet, A. Bottino, P. Angelino, R. Hatzky, T. Tran, B. McMillan, O. Sauter, K. Appert, Y. Idomura, and L. Villard, Comput. Phys. Commun. 177, 409 (2007).

[6] X. Lapillonne, Ph.D. thesis, EPFL, 2010.

[7] T. Görler, Ph.D. thesis, Universität Ulm, 2009.

[8] X. Lapillonne, B. McMillan, T. Görler, S. Brunner, T. Dannert, F. Jenko, F. Merz, and L. Villard (to be published).

[9] F. Jenko, W. Dorland, M. Kotschenreuther, and B. N. Rogers, Phys. Plasmas 7, 1904 (2000).

[10] X. Lapillonne, S. Brunner, T. Dannert, S. Jolliet, A. Marinoni, L. Villard, T. Görler, F. Jenko, and F. Merz, Phys. Plasmas 16, 032308 (2009).

[11] B. F. McMillan, S. Jolliet, T. M. Tran, L. Villard, A. Bottino, and P. Angelino, Phys. Plasmas 15, 052308 (2008).

[12] W. X. Wang, Z. Lin, W. M. Tang, W. W. Lee, S. Ethier, J. L. V. Lewandowski, G. Rewoldt, T.S. Hahm, and J. Manickam, Phys. Plasmas 13, 092505 (2006).

[13] Z. Lin and T. Hahm, Phys. Plasmas 11, 1099 (2004).

[14] T. S. Hahm, P. H. Diamond, Z. Lin, K. Itoh, and S.-I. Itoh, Plasma Phys. Controlled Fusion 46, A323 (2004).

[15] B.F. McMillan, S. Jolliet, T. M. Tran, L. Villard, A. Bottino, and P. Angelino, Phys. Plasmas 16, 022310 (2009). 